\title{
Multiple Breath Wash-out for early assessment of pulmonary complications in patients with Primary Antibody Deficiencies: an observational study in pediatric age
}

\section{Teresa Secchi}

Fondazione IRCCS Ca' Granda Ospedale Maggiore Policlinico

Lucia Augusta Baselli ( $\sim$ lucia.base@gmail.com )

Fondazione IRCCS Ca' Granda Ospedale Maggiore Policlinico https:// orcid.org/0000-0003-1171-1185

Maria Chiara Russo

Fondazione IRCCS Ca' Granda Ospedale Maggiore Policlinico Irene Borzani

Fondazione IRCCS Ca' Granda Ospedale Maggiore Policlinico

\section{Federica Carta}

Fondazione IRCCS Ca' Granda Ospedale Maggiore Policlinico

Amalia Lopopolo

Fondazione IRCCS Ca' Granda Ospedale Maggiore Policlinico

\section{Michaela Foà}

Fondazione IRCCS Ca' Granda Ospedale Maggiore Policlinico

\section{Adriano La Vecchia}

Fondazione IRCCS Ca' Granda Ospedale Maggiore Policlinico

\section{Carlo Virginio Agostoni}

Fondazione IRCCS Ca' Granda Ospedale Maggiore Policlinico

\section{Massino Agosti}

University of Insubria

\section{Rosa Maria Dellepiane}

Fondazione IRCCS Ca' Granda Ospedale Maggiore Policlinico

\section{Research Article}

Keywords: Human Inborn Errors of Immunity, Primary Antibody Deficiencies, Lung Clearance Index, Multiple Breath Wash-out, Chronic pulmonary disease, Respiratory Function

Posted Date: March 17th, 2021 
DOI: https://doi.org/10.21203/rs.3.rs-300039/v1

License: (c) (1) This work is licensed under a Creative Commons Attribution 4.0 International License. Read Full License 


\section{Abstract}

Purpose. In Primary Antibody Deficiencies (PADs), pulmonary complications are the main cause of morbidity, despite immunoglobulin substitutive therapy, antibiotic treatment of exacerbations and respiratory physiotherapy. Current recommendations for surveillance of PADs respiratory complications include the annual assessment of spirometry and the execution of chest High Resolution Computed Tomography (HRCT) every four years.

This study aims to evaluate the effectiveness of Lung Clearance Index $(\mathrm{LCl})$ as early marker of lung damage in patients with PADs. $\mathrm{LCl}$ is measured by Multiple Breath Wash-out, a non-invasive and highly specific test widely used in Cystic Fibrosis patients.

Methods. Pediatric patients with PADs ( $n=17,10, M, 7, F$, age range 5-15 years) underwent baseline assessment of lung involvement with chest HRCT, Spirometry and Multiple Breath nitrogen Wash-out. Among them, 13 patients were followed up to repeat HRCT after four years, while performing Pulmonary Function Tests annually. Their baseline and control LCI and FEV1 values were compared, taking HRCT as gold standard, using logistic regression analysis.

Results. LCI (OR 2.43 at baseline, OR 14.95 at control) has a stronger discriminating power between altered and normal HRCT rather than FEV1 (OR 0.82 at baseline, OR 1.91 at control).

Conclusion. Within the context of a limited sample size, $\mathrm{LCl}$ seems to be more predictive of HRCT alterations than FEV1 and more sensitive than HRCT in detecting ventilation inhomogeneity, in absence of bronchiectasis. A study of a larger cohort of pediatric patients followed longitudinally in adulthood is needed to challenge these findings.

\section{Introduction}

Primary Antibody Deficiencies (PADs), a heterogeneous spectrum of conditions characterized by marked reduction in blood levels of at least one main immunoglobulin (Ig) class, are the most representative diseases among all human inborn errors of immunity, accounting for about half of the cases of primary immunodeficiency [1-6].

Pulmonary diseases, and especially respiratory tract infections, are among the main causes of morbidity and mortality in patients with PADs, although their prognosis has been significantly improved by adequate immunoglobulin substitutive therapy, aggressive antibiotic therapy of sinopulmonary exacerbations and personalized respiratory physiotherapy programs. The presence of chronic lung injury at diagnosis represents the major predictor of mortality, while an early diagnosis and the timely start of $\mathrm{Ig}$ substitutive therapy, together with well-conducted respiratory physiotherapy, are predictors of positive outcomes for patients with PADs [7-11]. Detecting early signals of a worsening pulmonary function is therefore critical to prevent progression towards chronic lung disease. 
Respiratory tract infections can be relevant when occurring acutely, but their recurrence can also have long-term effects on the lung architecture, by remodeling airways. As result of repeated infectious and inflammatory episodes leading to destruction of airways and lung parenchyma with parallel decline in respiratory function, bronchiectasis are common in patients with PADs. Chest High Resolution Computed Tomography (HRCT) is currently the gold standard for the detection and characterization of bronchiectasis and respiratory complications $[10,12]$.

The current AIEOP/IPINet recommendations (AIEOP, Italian Association of Pediatric Hematology Oncology and IPINet, Italian Primary Immunodeficiencies Network) for the surveillance of respiratory complications in PADs recommend the annual evaluation of lung function with spirometry, associated with chest HRCT every four years in otherwise clinically stable patients. Spirometry has to be carried out in well-being or at least three weeks after recovery from an episode of respiratory exacerbation, while HRCT must be anticipated in case of an episode of severe respiratory exacerbation requiring hospitalization or if the patient is not clinically healthy and possibly performed at least three weeks after recovery [13].

Spirometry detects dynamic lung volumes by measuring the movement of the inhaled and exhaled air during respiratory maneuvers and by expressing it as a function of time. One of the parameters detected during spirometry is the Forced Expiratory Volume at 1 second (FEV1), the volume of air exhaled in the first second of a forced exhalation. FEV1 represents the index parameter of the bronchial obstruction degree, as well as being a good indicator of airway resistance. FEV1 is commonly used as the primary tool in monitoring patients with bronchiectasis $[8,9,14]$. However, both in literature and in clinical practice, there is increased evidence that FEV1 is poorly sensitive in identifying early lung damage. Most clinical trials performed on patients with bronchiectasis showed that the initial pulmonary impairment identifiable through chest HRCT was not detectable by FEV1, which was normal in most patients [15-18]. On the other hand, exposing PADs patients to radiation increase the risk of neoplasms in the long term. Due to the underlying disease, PADs patients have a greater risk of neoplastic disease compared to the general population. Primarily in pediatric PADs patients, minimizing radiation exposure is mandatory, also considering the improvement in their life expectancy of the last years [19].

Multiple Breath Inert Gas Wash-out (MBW) assesses static lung volumes and inhomogeneity of lung ventilation during tidal breathing providing, as a functional parameter, the Lung Clearance Index $(\mathrm{LCl})$, the number of lung volumes required to complete the wash-out of a tracer gas inhaled or already present in the lungs. For the execution of this non-invasive respiratory test, only minimal cooperation is required from the patient, so that it is potentially applicable to all ages. MBW has therefore assumed increasing clinical importance in the follow-up of chronic pulmonary disease in pediatric patients, such as those with Cystic Fibrosis (CF) [20-22].

On the whole, observations in CF patients suggest that the MBW determination may provide a repeatable and highly sensitive measure of lung function in patients with bronchiectasis, particularly those with preserved spirometry [23-25]. 
This study therefore aims to evaluate the effectiveness of $\mathrm{LCl}$, measured by MBW, as early marker of lung damage and to evaluate its trend over time during the follow-up of respiratory complications in patients with PADs.

\section{Methods}

\section{Trial design and participants}

A four-year spontaneous/non-profit single-center prospective observational design has been conducted from December, 2015, up to January, 2020. The study was approved by the Ethics Committee (EC) of the IRCCS Fondazione Ca' Granda (EC approval number: 55_2018bis).

The primary goal of the study was to evaluate the effectiveness of $\mathrm{LCl}$, measured by nitrogen MBW, as early marker of lung damage in patients with PADs, even in the absence of bronchiectasis. Secondarily the study aimed to evaluate the overtime trend of $\mathrm{LCl}$ in patients with PADs during follow-up of respiratory complications, verifying the usefulness of MBW in monitoring the respiratory function and in the early highlighting of the onset of pulmonary complications.

Inclusion criteria in the study were: patients suffering from PADs diagnosed according to the European Society for Immunodeficiencies (ESID) criteria [26]; patients in follow-up of the respiratory complications according to the AIEOP/IPINet recommendations [13]; availability of chest HRCT performed in well-being at least once in the last four years; age > five years; compliance with the study protocol and ability in performing Pulmonary Function Tests (PFTs) according to the criteria of reliability. Exclusion criteria were considered age < five years; patients not cooperating and/or not able to carry out the test correctly.

Seventeen patients ( 10 males and 7 females, age range between 5.7 and 14.8 years) with PADs were involved in the study: 11 (6 males and 5 females) with Common Variable Immunodeficiency (CVID), 2 males with X-linked Agammaglobulinemia (XLA) and 1 male with Autosomal Recessive Agammaglobulinemia (ARA), 2 female patients with Selective IgA Deficiency (SIgAD) with respiratory complications and 1 male with Hyper IgM Syndrome (HIGM). 12 out of the 17 patients (8 CVID, 2 XLA, 1 ARA and $1 \mathrm{HIGM}$ ) were undergoing Ig substitutive therapy at the time of the study, all with adequate preinfusion IgG levels (median $915 \mathrm{mg} / \mathrm{dl} ;$ IQR 872-964). All patients were also following a personalized respiratory physiotherapy program. The median age of symptoms onset in all patients involved was 1.5 years (IQR 1.0-3.3). The median age at diagnosis was 6.6 years (IQR 4.3-9.8). The median diagnosis delay was 3.2 years (IQR 1.3-6.7). The patients median age at baseline was 10.7 years (IQR 8.5-12.6). The median duration of follow-up for this study was 4.0 years (IQR 3.2-4.0) (Table 1).

\section{Intervention}

According to the study design, MBW with $\mathrm{LCl}$ determination has been included in the standard follow-up of patients with PADs in order to monitor the progression of respiratory complications. At the time of enrollment each patient has been subjected to a global baseline assessment of lung involvement by 
performing spirometry, nitrogen MBW and chest HRCT. For 13 out of the 17 patients with stable conditions the Pulmonary Function Tests (spirometry and MBW), performed annually, have been compared with chest HRCT, performed every four years. For the other 4 patients, enrolled later, the followup was too limited to check repeated chest HRCT and comparison has been possible only at baseline time.

HRCT scan studies were scored adopting the Bhalla score [27] in a random order by a pediatric radiologist and a pediatric pneumologist, blinded to patients' identities and clinical information, respectively. Readers evaluated each examination independently and reporting in consensus [28,29].

The Bhalla score [27] is a morphological scoring system usually applied to monitor pulmonary disease progression and stage of chronic broncho-pneumopathies. Bhalla Score can vary between 0 and 25 and includes presence, severity and extent of different pulmonary alterations, including bronchiectasis, peribronchial thickening, mucous plugging, sacculation/abscesses, bullae, emphysema and collapse/consolidation [12,30].

Each MBW test consists of a wash-in and a wash-out phase. This respiratory test is feasible in two ways using an extrinsic inert tracer gas, inhaled until equilibrium is reached and then eliminated by breathing ambient air, or by inhaling $100 \%$ oxygen for the wash-out of nitrogen, an intrinsic inert gas normally present in the airways, respectively. In our survey $100 \%$ oxygen was used for the nitrogen wash-out in order to increase sensitivity in identifying abnormalities being nitrogen present in all areas of the lung $[20,21]$.

Parameters derived from MBW are obtained on the analysis of the breath-by-breath concentrations and volumes of gas. The LCl may be considered the most reliable MBW parameter since it reflects the inhomogeneity of pulmonary ventilation and is defined as the number of lung turnovers required to washout the tracer gas up to $2.5 \%$ of its initial concentration. In a healthy person, this takes about 5-7 turnovers. According to a 2011 review by Fuchs and Gappa [15], which reports the most relevant studies conducted to define LCl normal values and Upper Limit of Normal (ULN) in pediatric age, $\mathrm{LCl}$ is constant during childhood and independent of age, weight, height and gender during adolescence. The value of 7.00 is reported as ULN for $\mathrm{LCl}$ in childhood. In case of ventilatory inhomogeneity, $\mathrm{LCl}$ increases with the number of turn-over needed for the tracer gas to be eliminated from the lungs $[15,20,22,31]$.

\section{Statistical methods}

The median age of the 17 patients at the time of baseline HRCT was 10.7 years (IQR 8.5-12.6), while the median age at the HRCT control after four years was 14.8 years (IQR 13.7-16.5) (Table 1).

Parameters of the respiratory function tests considered for the study were FEV1 as a percentage of the predicted value ( $\mathrm{FEV} 1 \% \mathrm{mis} / \mathrm{T}$ ) and $\mathrm{LCl}$ at $2.5 \%$ of the gas initial concentration ( $\mathrm{LCl} 2.5 \%)$ calculated with nitrogen MBW, respectively. 
Regarding the cross-sectional evaluation, $\mathrm{LCl}$ and FEV1 parameters were compared, taking the HRCT as gold standard, evaluated by Bhalla score. Given the non-normal and asymmetrical distribution of the Bhalla score, in order to better analyze the data, HRCT was transformed into a dichotomous variable and statistical analyzes were accordingly performed by splitting the patients in two groups. Accordingly, HRCT has been considered normal (N-CT) for patients with Bhalla score $=0$, while it has been considered altered $(\mathrm{A}-\mathrm{CT})$ for patients with Bhalla score $>0$. Fisher's exact test has been employed to test the dependency on gender, while age, FEV1 and LCl were compared using the Wilcoxon-Mann-Whitney test. Univariate and multivariate (adjusting for gender and age) logistic regression analyses were used to study the association between FEV1, LCl and N-CT/A-CT.

\section{Results}

Spirometry turned out to be normal or slightly altered in all enrolled patients. FEV1 below $80 \%$ of the predicted values, considered as Lower Limit of Normal (LLN) [14], have been found 4 times out of 56 measurements $(7.14 \%$ of cases). In two of these cases spirometry was repeated in otherwise stable patients three months later, following an intensification of the respiratory physiotherapy program, with return to normal basal values. Median FEV1 value observed at the baseline time was 105.2\% (IQR 93.1117.1) and 97.2\% (IQR 88.0-102.4) at the time of the second HRCT (Figure 1).

Although spirometry was always normal or just mildly altered, HRCT was found altered in 8/17 (47\%) patients at baseline, and in 6/13 (46\%) patients at follow-up after four years. At baseline 3 A-TC patients (18\%) were reported with moderately altered Bhalla scores (median Bhalla score 7.0, IQR 7.0-8.5) and 5 ATC patients (29\%) with slightly altered Bhalla scores (median Bhalla score 1.0, IQR 1.0-3.0). At the time of control 2 A-TC patients (15\%) were reported with moderately altered Bhalla scores (median Bhalla score 6.5, IQR 6.25-6.75) and 4 A-TC patients (31\%) with slightly altered Bhalla scores (median Bhalla score 1.0, IQR 1.0-1.75) (Figure 2). The HRCT images of patients with moderately altered Bhalla scores showed the presence of bronchiectasis with thickened walls and other pathological findings such as collapse and consolidation, peri-bronchial thickening and mucus plugs. Regardless of the severity and extent of bronchiectasis, both of which were mild in 2 out of 3 patients, the severity of the pulmonary picture is determined by the involvement of the more distal bronchioles.

The lung function study was completed through the evaluation of ventilatory inhomogeneity by nitrogen MBW with $\mathrm{LCl}$ detection at $2.5 \%$ of the initial concentration of tracer gas. $\mathrm{LCl}$ values $>7$ were found in more than $50 \%$ patients. The median $\mathrm{LCl}$ value at the baseline time was 7.3 (IQR 6.74-7.92) and 7.4 (IQR 7.05-7.79) at the time of control corresponding to the second HRCT (Figure 3).

At baseline and at the time of radiological control, LCl and FEV1 parameters were compared taking HRCT as gold standard. No significant differences emerged in terms of the distributions of FEV1 and LCI between patients with normal or altered HRCT (Figure 4 and Figure 5). The comparison of ages of the two groups of patients was examined by Wilcoxon-Mann-Whitney test ( $p$-value 0.04 ) with younger patients showing a more altered HRCT (Figure 6). 
After standardizing FEV1 and LCl values, univariate and multivariate (including age and gender as independent variables) logistic regression analyses were performed, using N-CT/A-CT as dependent variable. FEV1 at baseline showed a weak negative association with A-CT (age and gender adjusted, OR = 0.82 ). On the contrary, $\mathrm{LCl}$ at baseline showed a positive association (age and gender adjusted, $\mathrm{OR}=$ 2.43). The same analyses were performed with the sample of 13 patients at the 4 years later HRTC control. Accordingly, FEV1 after four years showed a weak positive association with A-CT (age and gender adjusted, $\mathrm{OR}=1.91$ ), while $\mathrm{LCl}$ showed a stronger positive association after 4 years (age and gender adjusted, $O R=14.95$ ).

\section{Discussion}

According to the literature, the follow-up of lung involvement in patients with human inborn errors of immunity requires improvements by introducing innovative tests aimed at the early detection of the onset of lung damage, thus offering the opportunity for a change in the clinical management of patients. Hence the need to study a non-invasive test, easily to perform even by youngest patients, for an earlier identification of the decline in respiratory function at an early stage.

The present survey describes the first study evaluating $\mathrm{LCl}$ in the pediatric population of patients with inborn errors of immunity, inclusive of 17 patients suffering from different types of PADs and sharing similar clinical pictures concerning lung involvement.

Our observations suggest that LCl might be a parameter with higher sensitivity when compared with FEV1 in detecting lung abnormalities, even in clinically stable situations. Except in one single case, the Pulmonary Function Tests were always performed in stable conditions and no patient had experienced significant respiratory exacerbations before performing the MBW.

Although all patients presented with various degrees of airway disease, $82 \%$ of patients with altered HRCT at baseline and/or control had FEV1 values within the normal range, despite their $\mathrm{LCl}$ values were significantly altered in $64 \%$ of cases. Among patients with normal HRCT at baseline or at control, $91 \%$ had FEV1 values within the normal range, but $82 \%$ showed slightly altered LCl values. Nevertheless, alterations in $\mathrm{LCl}$ found in cases of normal pulmonary HRCT were milder than those found in patients with impaired chest HRCT.

A close clinical and instrumental monitoring over time is particularly indicated for patients with normal pulmonary HRCT and increased $\mathrm{LCl}$ to check ventilatory inhomogeneity as indicator of initial decline in lung function. A worsening of PFTs in patients with PADs, in particular LCl measured by MBW, should therefore represent an alarming sign indicating the need to introduce new interventions such as strengthening clinical check-up, implementation of respiratory physiotherapy, re-evaluation of Ig substitutive therapy, introduction of anti-inflammatory agents and more aggressive antibiotic therapy of infectious exacerbations. 
A major limit of this study is the small sample size, limiting the power of the study. Therefore, our results should be interpreted with care. Nevertheless, the results from our monocentric setting suggest the need to check in larger groups the higher predictive value of an increase in $\mathrm{LCl}$, compared to a reduction in FEV1, in evaluating alterations of pulmonary HRCT. While gender seems to have limited influence, age has a stronger impact on results, with younger patients showing a more altered HRCT. The age difference between the patients with normal or altered HRCT is likely due to differences in the severity of the underlying disease. In fact, patients in study had different forms of immunodeficiencies, with heterogeneous clinical presentations. Indeed, most patients with altered chest HRCT have a more severe disease expression, with an earlier onset of symptoms, while patients with normal HRCT generally have a less severe disease expression and a milder severity of the symptoms on the whole.

Another limitation of the study is represented by the length of the observational period. Accordingly, a follow-up of four years may still be temporarily limited to catch clinically relevant radiological changes. Furthermore, four patients were enrolled during the study just at the initial phase of their follow up. In any case, the relative stability of their clinical picture may support the validity of the surveillance and the personalized respiratory physiotherapy adopted.

Carrying out PFTs and HRCT on the same day was hard to organize for practical reasons. Therefore, we had to account for the time between HRCT and PFR for each patient, at the baseline and at the time of control, respectively. However, the assessments were always performed in well-being states and the statistical analysis did not demonstrate the influence on radiological results of the between-date intervals.

$\mathrm{LCl}$ measured by MBW therefore appears a parameter with higher sensitivity to predict the presence of anatomo-functional abnormalities as displayed on chest HRCT, in comparison with a reduced FEV1. Indeed, at least within a pediatric setting, LCl seems to be able to detect alveolar inhomogeneity (with the functional derivable correlates), even in the absence of x-rays detectable bronchiectasis and alterations at spirometry. $\mathrm{LCl}$ could therefore integrate the Bhalla score, which does not include alveolar inhomogeneity, and its periodical assessment could help the early detection of a worsening of respiratory function, before the occurrence of alterations at radiological examinations and spirometry.

Further studies on larger cohorts of pediatric PADs patients followed longitudinally up to adulthood within a multicentric context are required in order to validate the usefulness of MBW in this population. An adequate sample size would also allow for a comparison between subjects with the same type of human inborn error of immunity (considering the different forms of PADs) and a consequent more detailed analysis of the different subgroups of patients, according to the emerging concepts of precision medicine.

\section{Abbreviations}

- A-CT, Altered HRCT 
- AIEOP, Italian Association of Pediatric Hematology Oncology

- ARA, Autosomal Recessive Agammaglobulinemia

- CF, Cystic Fibrosis

- CVID, Common Variable Immunodeficiency

- EC, Ethics Committee

- ESID, European Society for Immunodeficiencies

- FEV1, Forced Expiratory Volume at 1 second

- HIGM, Hyper IgM Syndrome

- HRCT, High Resolution Computed Tomography

- Ig, immunoglobulin

- IPINet, Italian Primary Immunodeficiencies Network

- IQR, Interquartile Range

- LCl, Lung Clearance Index

- LLN, Lower Limit of Normal

- MBW, Multiple Breath Wash-Out

- MR, Moment Ratio

- N-CT, Normal HRCT

- OR, Odds Ratio

- PADs, Primary Antibody Deficiencies

- PFTs, Pulmonary Function Tests

- SD, Standard Deviation

- SIgAD, Selective IgA Deficiency

- ULN, Upper Limit of Normal

- XLA, X-Linked Agammaglobulinemia

\section{Declarations}

Funding: partly supported by a funding of the Italian Ministry of Health for IRCCS Institutes.

Conflicts of interest/Competing interests: The authors declare that they have no conflict of interest.

Availability of data and material: not applicable

Code availability: not applicable

Authors' contribution: All authors contributed to the study conception and design. Material preparation and data collection were performed by Teresa Secchi, data analysis was performed by Adriano La 
Vecchia. The first draft of the manuscript was written by Teresa Secchi and all authors commented on previous versions of the manuscript. All authors read and approved the final manuscript.

Ethics approval: The study protocol was approved by the local Ethic Committee. Ethics Committee approval number: 55_2018bis

Consent to participate/publication: written informed consent was obtained from all the subject participating in the study.

\section{Acknowledgement}

We wish to thank all the patients and family members that participated in the study.

\section{References}

1. Plebani A, Badolato R, Soresina A, Lougaris V. Immunodeficienze primitive. In: Società Editrice Esculapio, editor. Zuccotti, GV Manuale di Pediatria - La Pratica Clinica. Seconda Ed. Milano; 2016. p. 250-64.

2. Tangye SG, Al-Herz W, Bousfiha A, Chatila T, Cunningham-Rundles C, Etzioni A, et al. Human Inborn Errors of Immunity: 2019 Update on the Classification from the International Union of Immunological Societies Expert Committee. J Clin Immunol. 2020 Jan 1;

3. Bousfiha A, Jeddane L, Picard C, Al-Herz W, Ailal F, Chatila T, et al. Human Inborn Errors of Immunity: 2019 Update of the IUIS Phenotypical Classification. J Clin Immunol. 2020 Jan 1;40(1):66-81.

4. Picard C, Bobby Gaspar H, Al-Herz W, Bousfiha A, Casanova JL, Chatila T, et al. International Union of Immunological Societies: 2017 Primary Immunodeficiency Diseases Committee Report on Inborn Errors of Immunity. J Clin Immunol. 2018 Jan 1;38(1):96-128.

5. The ESID Registry [Internet]. [cited 2020 Nov 3]. Available from: https://cci-esid-reg.uniklinikfreiburg.de/EERS/login/auth

6. Wekell P, Hertting $\mathrm{O}$, Holmgren D, Fasth A. Fifteen-minute consultation: Recognising primary immune deficiencies in children. Arch Dis Child Educ Pract Ed. 2019 Oct 1;104(5):235-43.

7. Mahdaviani SA, Darougar S, Mansouri D, Tashayoie-Nejad S, Movahedi M, Aghdam KR, et al. Pulmonary complications of predominantly antibody immunodeficiencies in a tertiary lung center. Interv Med Appl Sci. 2019 Mar;11(1):1-7.

8. Schütz K, Alecsandru D, Grimbacher B, Haddock J, Bruining A, Driessen G, et al. Imaging of Bronchial Pathology in Antibody Deficiency: Data from the European Chest CT Group. J Clin Immunol. 2019 Jan 15;39(1):45-54.

9. Jolles S, Sánchez-Ramón S, Quinti I, Soler-Palacín P, Agostini C, Florkin B, et al. Screening protocols to monitor respiratory status in primary immunodeficiency disease: findings from a European survey and subclinical infection working group. Clin Exp Immunol. 2017 Nov 1;190(2):226-34. 
10. Cinetto F, Scarpa R, Rattazzi M, Agostini C. The broad spectrum of lung diseases in primary antibody deficiencies. Eur Respir Rev. 2018 Sep 30;27(149):180019.

11. Schussler E, Beasley MB, Maglione PJ. Lung Disease in Primary Antibody Deficiencies. J Allergy Clin Immunol Pract. 2016 Nov;4(6):1039-52.

12. Başaran AE, Başaran A, Maslak iC, Arslan G, Bingöl A. Evaluation of noncystic fibrosis bronchiectasis using clinical and radiological scorings in children. Turkish Thorac J. 2018 Oct 1;19(4):159-64.

13. Ugazio A, Pietrogrande MC, Rusconi F, Cazzola GA, Plebani A, Soresina A, et al. Addendum alle raccomandazioni per I'XLA - Complicanze respiratorie nei deficit dell'immunità umorale [Internet]. 2004 [cited 2020 May 11]. p. 21. Available from: https://www.aieop.org/web/operatorisanitari/gruppi-di-lavoro/immunodeficienze/

14. Graham BL, Steenbruggen I, Barjaktarevic IZ, Cooper BG, Hall GL, Hallstrand TS, et al. Standardization of spirometry 2019 update an official American Thoracic Society and European Respiratory Society technical statement. Vol. 200, American Journal of Respiratory and Critical Care Medicine. American Thoracic Society; 2019. p. E70-88.

15. Fuchs SI, Gappa M. Lung clearance index: Clinical and research applications in children. Vol. 12, Paediatric Respiratory Reviews. 2011. p. 264-70.

16. Lombardi E, Gambazza S, Pradal U, Braggion C. Lung clearance index in subjects with cystic fibrosis in Italy. Vol. 45, Italian Journal of Pediatrics. BioMed Central Ltd.; 2019. p. 56.

17. Chelabi R, Soumagne T, Guillien A, Puyraveau M, Degano B. In cystic fibrosis, lung clearance index is sensitive to detecting abnormalities appearing at exercise in children with normal spirometry. Respir Physiol Neurobiol. 2018 Jan 1;247:9-11.

18. Stanojevic S, Ratjen F. Physiologic endpoints for clinical studies for cystic fibrosis. Vol. 15, Journal of Cystic Fibrosis. Elsevier B.V.; 2016. p. 416-23.

19. Mortaz E, Tabarsi P, Mansouri D, Khosravi A, Garssen J, Velayati A, et al. Cancers related to immunodeficiencies: Update and perspectives. Vol. 7, Frontiers in Immunology. Frontiers Media S.A.; 2016.

20. Usemann J, Yammine S, Singer F, Latzin P. Inert gas washout: background and application in various lung diseases. Vol. 147, Swiss Medical Weekly. EMH Swiss Medical Publishers Ltd.; 2017.

21. Fretzayas A, Douros K, Moustaki M, Loukou I. Applications of lung clearance index in monitoring children with cystic fibrosis. World J Clin Pediatr. 2019 Apr 9;8(2):15-22.

22. Arianto L, Hallas HW, Stokholm J, Bønnelykke K, Bisgaard H, Chawes BL. Sensitivity of multiple breath washout to detect mild-to-moderate asthma in adolescence. J Allergy Clin Immunol Pract. 2019 Jul 1;7(6):2052-2054.e5.

23. Kasi AS, Wee CP, Keens TG, Salinas DB. Abnormal Lung Clearance Index in Cystic Fibrosis Screen Positive, Inconclusive Diagnosis (CFSPID) Children with Otherwise Normal FEV1. Lung. 2020 Feb 1;198(1):163-7.

24. Perrem $L$, Rayment $J H$, Ratjen $F$. The lung clearance index as a monitoring tool in cystic fibrosis: ready for the clinic? Vol. 24, Current opinion in pulmonary medicine. NLM (Medline); 2018. p. 579- 
85.

25. Ramsey KA, Rosenow T, Turkovic L, Skoric B, Banton G, Adams AM, et al. Lung clearance index and structural lung disease on computed tomography in early cystic fibrosis. Am J Respir Crit Care Med. 2016 Jan 1;193(1):60-7.

26. ESID ES for I. ESID - European Society for Immunodeficiencies [Internet]. [cited 2020 Apr 11]. Available from: https://esid.org/Working-Parties/Registry-Working-Party/Diagnosis-criteria

27. Bhalla M, Turcios N, Aponte V, Jenkins M, Leitman BS, McCauley DI, et al. Cystic fibrosis: scoring system with thin-section CT. Radiology. 1991 Jun;179(3):783-8.

28. Milito C, Pulvirenti F, Serra G, Valente M, Pesce AM, Granata G, et al. Lung Magnetic Resonance Imaging with Diffusion Weighted Imaging Provides Regional Structural as well as Functional Information Without Radiation Exposure in Primary Antibody Deficiencies. J Clin Immunol. 2015 Jul 25;35(5):491-500.

29. Haidopoulou K, Calder A, Jones A, Jaffe A, Sonnappa S. Bronchiectasis secondary to primary immunodeficiency in children: Longitudinal changes in structure and function. Pediatr Pulmonol. 2009 Jul;44(7):669-75.

30. Reisi M, Azizi G, Kiaee F, Masiha F, Shirzadi R, Momen T, et al. Evaluation of pulmonary complications in patients with primary immunodeficiency disorders. Eur Ann Allergy Clin Immunol. 2017:49(3):122-8.

31. Kent L, Reix P, Innes JA, Zielen S, Le Bourgeois M, Braggion C, et al. Lung clearance index: Evidence for use in clinical trials in cystic fibrosis. Vol. 13, Journal of Cystic Fibrosis. 2014. p. 123-38.

\section{Tables}

Table 1. Clinical and demographic characteristics of the population in study. 


\begin{tabular}{|lllll|}
\hline Variable & $\mathrm{N}(\%)$ & Mean (SD) & Median (IQR) & Range \\
\hline $\begin{array}{l}\text { Age of symptoms onset } \\
\text { (years) }\end{array}$ & $17(100 \%)$ & $2.7(3.1)$ & $1.5(1.0-3.3)$ & $0.0-11.6$ \\
\hline $\begin{array}{l}\text { Age at diagnosis } \\
\text { (years) }\end{array}$ & $17(100 \%)$ & $7.0(4.2)$ & $6.6(4.3-9.8)$ & $0.7-14.8$ \\
\hline $\begin{array}{l}\text { Diagnosis delay } \\
\text { (years) }\end{array}$ & $17(100 \%)$ & $4.3(3.8)$ & $3.2(1.3-6.7)$ & $0.4-11.9$ \\
\hline $\begin{array}{l}\text { Age at baseline HRCT } \\
\text { (years) }\end{array}$ & $17(100 \%)$ & $10.4(3.0)$ & $10.7(8.5-12.6)$ & $5.7-14.8$ \\
$\begin{array}{l}\text { Age at HRCT control } \\
\text { (years) }\end{array}$ & $13(76 \%)$ & $14.7(2.8)$ & $14.8(13.7-16.5)$ & $9.1-18.1$ \\
$\begin{array}{l}\text { Duration of follow-up } \\
\text { (years) }\end{array}$ & $17(100 \%)$ & $3.4(1.2)$ & $4.0(3.2-4.0)$ & $0.4-4.9$ \\
\hline $\begin{array}{l}\text { Pre-infusion IgG levels } \\
\text { (mg/dl) }\end{array}$ & $12(71 \%)$ & $910(107)$ & $915(872-964)$ & $716-1080$ \\
\hline
\end{tabular}

SD, Standard Deviation; IQR, Interquartile Range; IgG, immunoglobulin G.

\section{Figures}



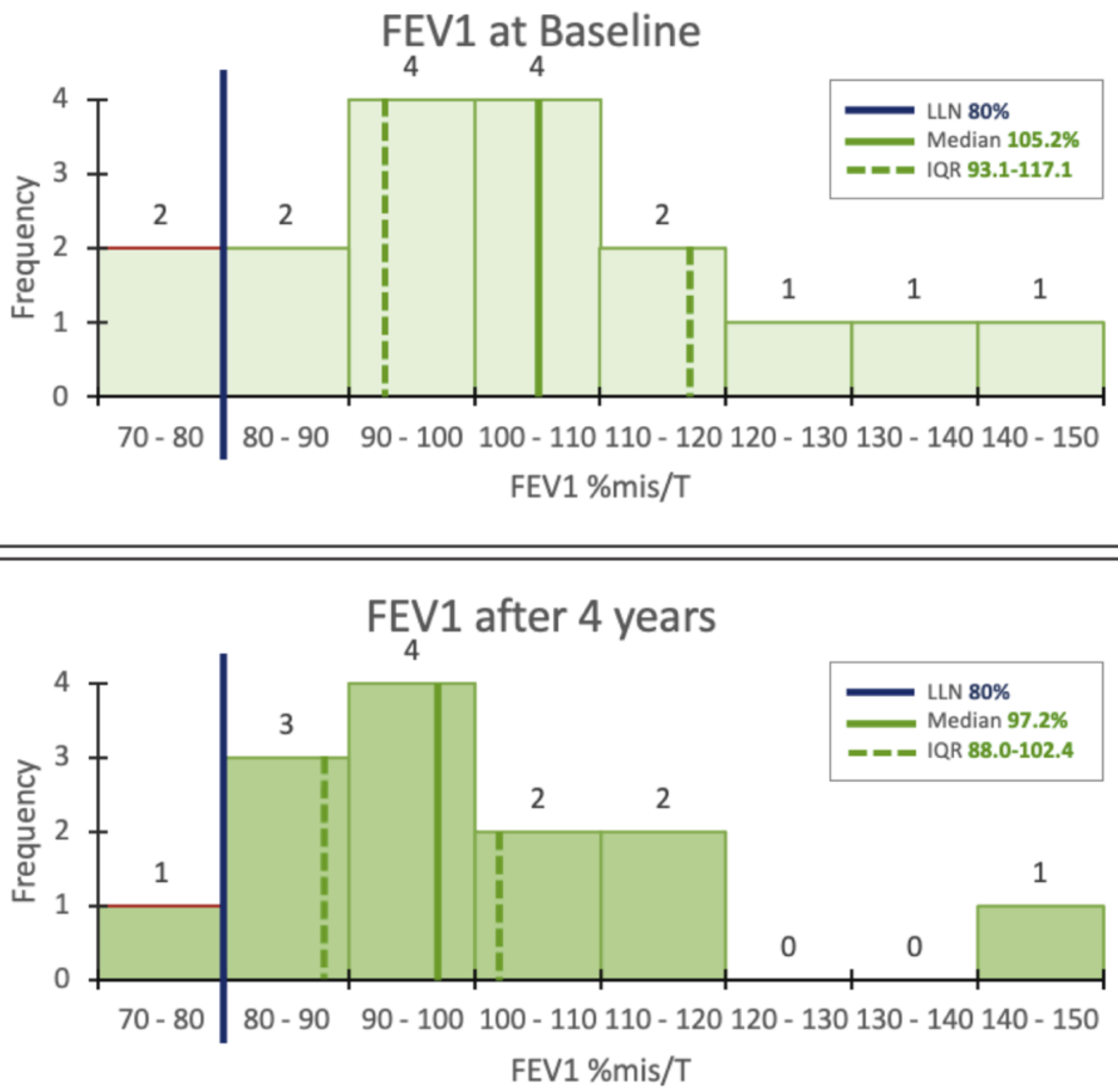

Figure 1

Frequency distribution of FEV1 (percentages values compared to the predicted) at baseline (top) and at the HRCT control (below). Blue line at the lower limit of normal, green lines at the FEV1 \% mis/T quartiles values. FEV1 \%mis/T, Forced Expiratory Volume at 1 second percentage values compared to the predicted; LLN, Lower Limit of Normal; IQR, Interquartile Range. 


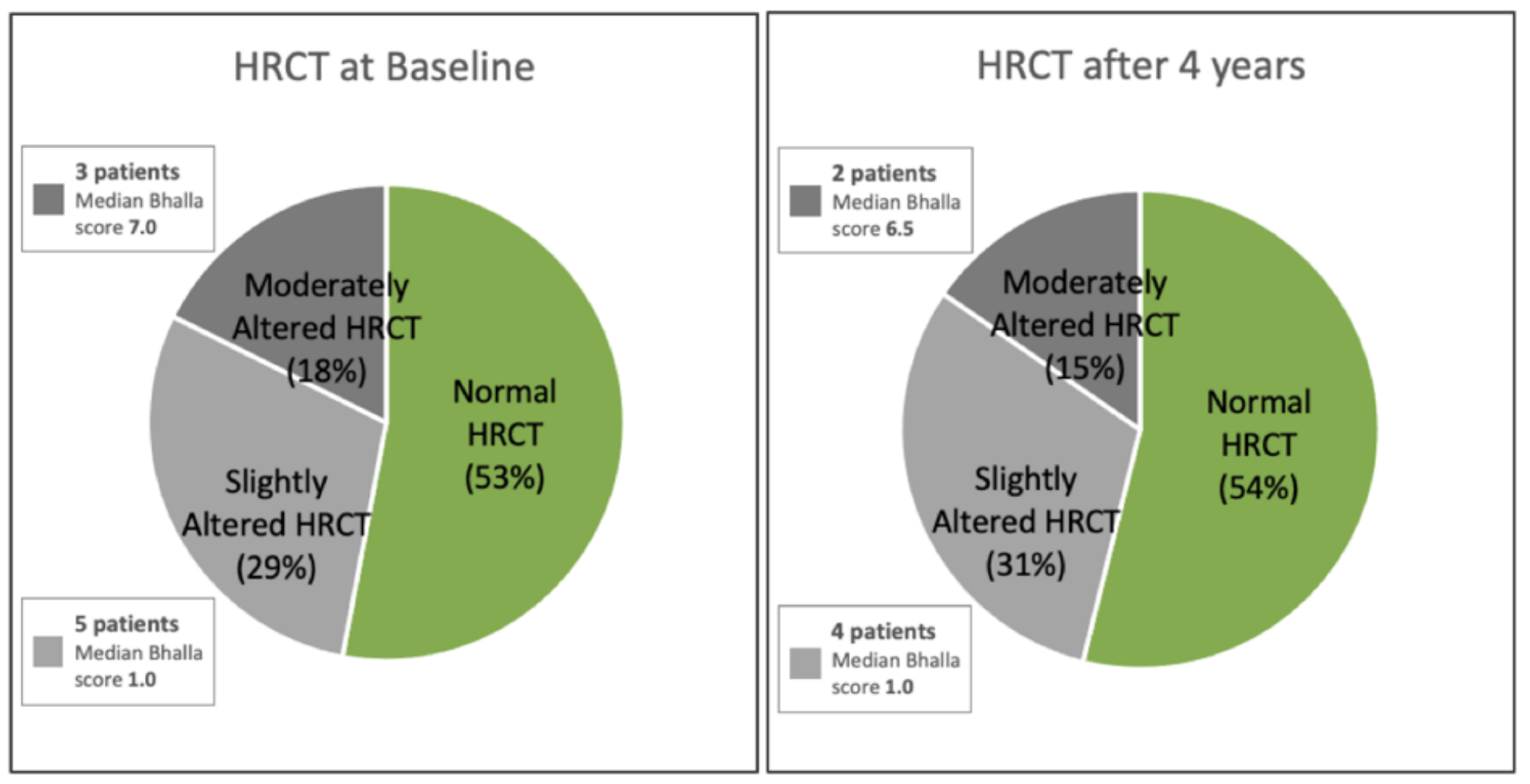

\section{Figure 2}

Proportion of patients with normal (Bhalla score 0 ), slightly altered (Bhalla score $\leq 4$, single values $\leq 1$, indicative of mild alterations) or moderately altered (Bhalla score $>4$, altered parameters $>1$, indicative of moderate or severe alterations) HRCT at the baseline (left) and at the HRCT control (right). HRCT, High Resolution Computed Tomography. 


\section{$\mathrm{LCl}$ at Baseline}

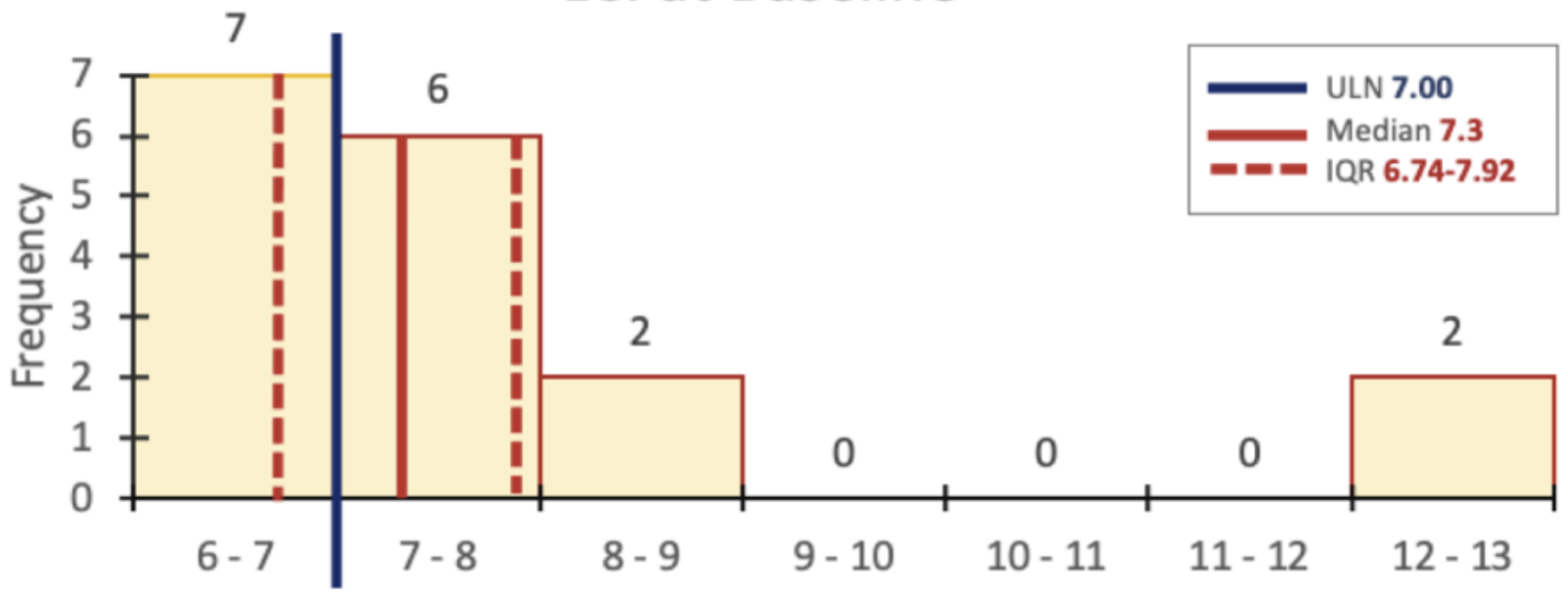

$\mathrm{LCl} 2.5 \%$

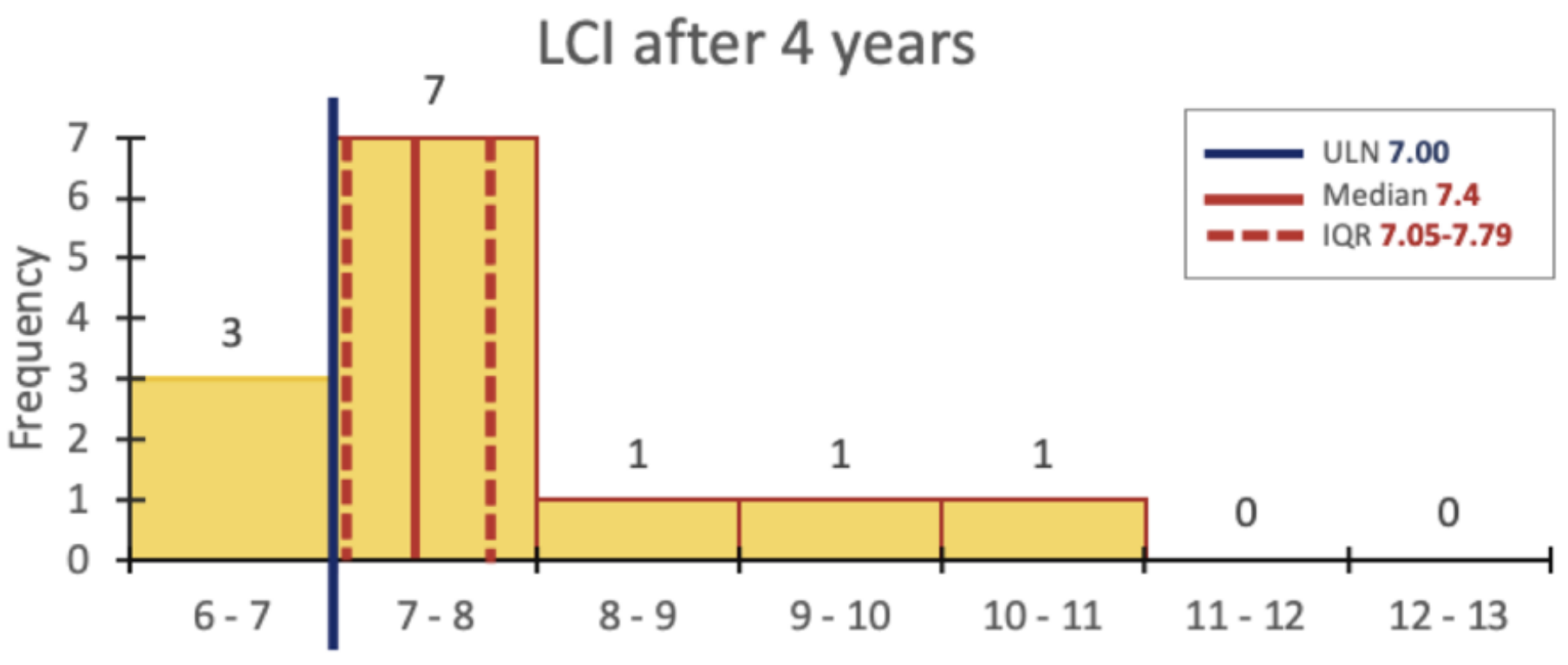

$\mathrm{LCl} 2.5 \%$

Figure 3

Frequency distribution of $\mathrm{LCl}$ at $2.5 \%$ of gas initial concentration at baseline (top) and at the HRCT control (below). Blue line at the upper limit of normal, red lines at the $\mathrm{LCl} 2.5 \%$ quartiles values. $\mathrm{LCI} 2.5 \%$, Lung Clearance Index at 2.5\% of the gas initial concentration; ULN, Upper Limit of Normal; IQR, Interquartile Range. 


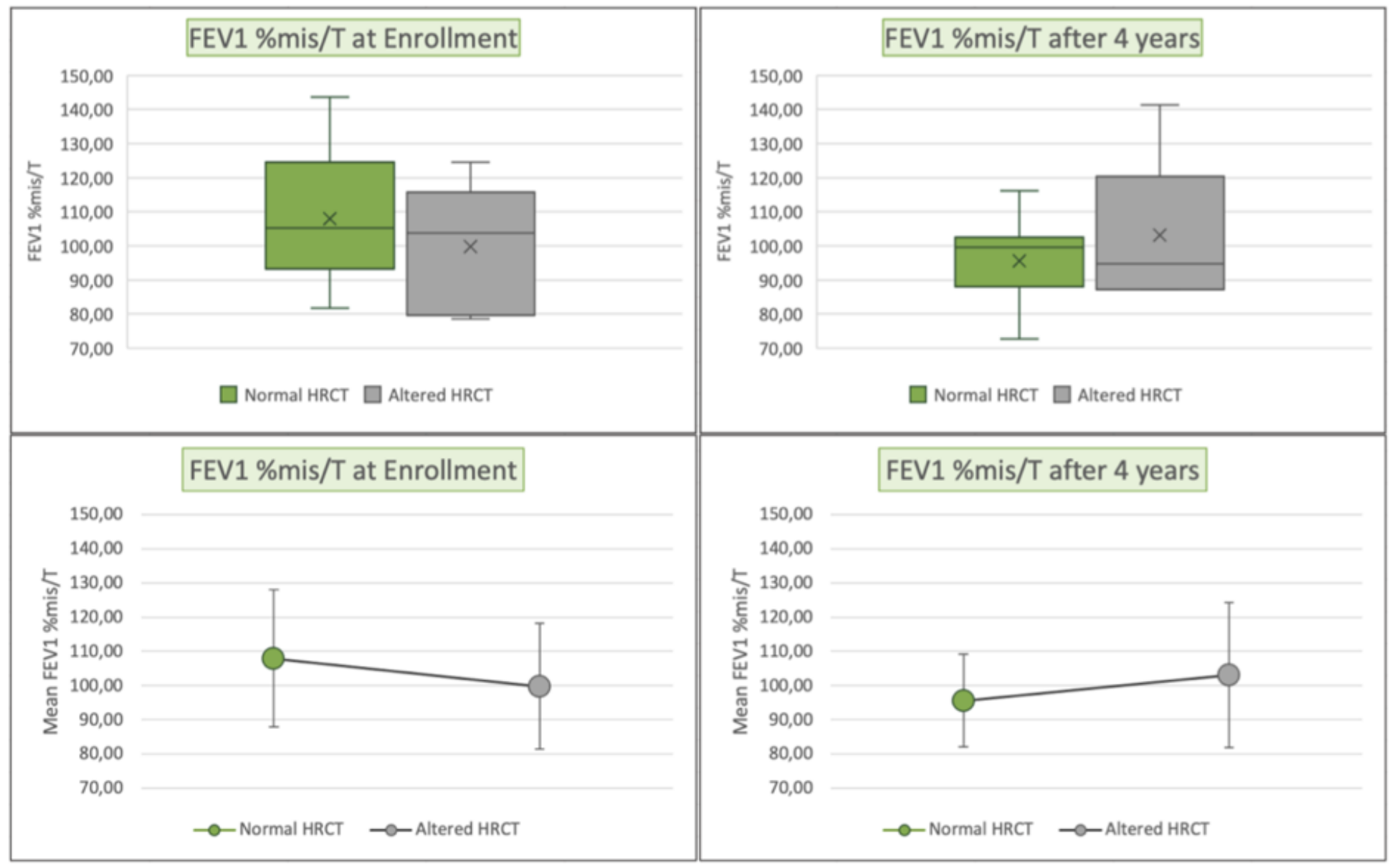

\section{Figure 4}

Box plots (above), Mean and Standard Deviation (below) of FEV1 (percentages values compared to the predicted) respectively for patients with normal HRCT (green) or altered HRCT (gray), at enrollment (left) and at the HRCT control (right). FEV1 \% mis/T, Forced Expiratory Volume at 1 second percentage values compared to the predicted; HRCT, High Resolution Computed Tomography. 


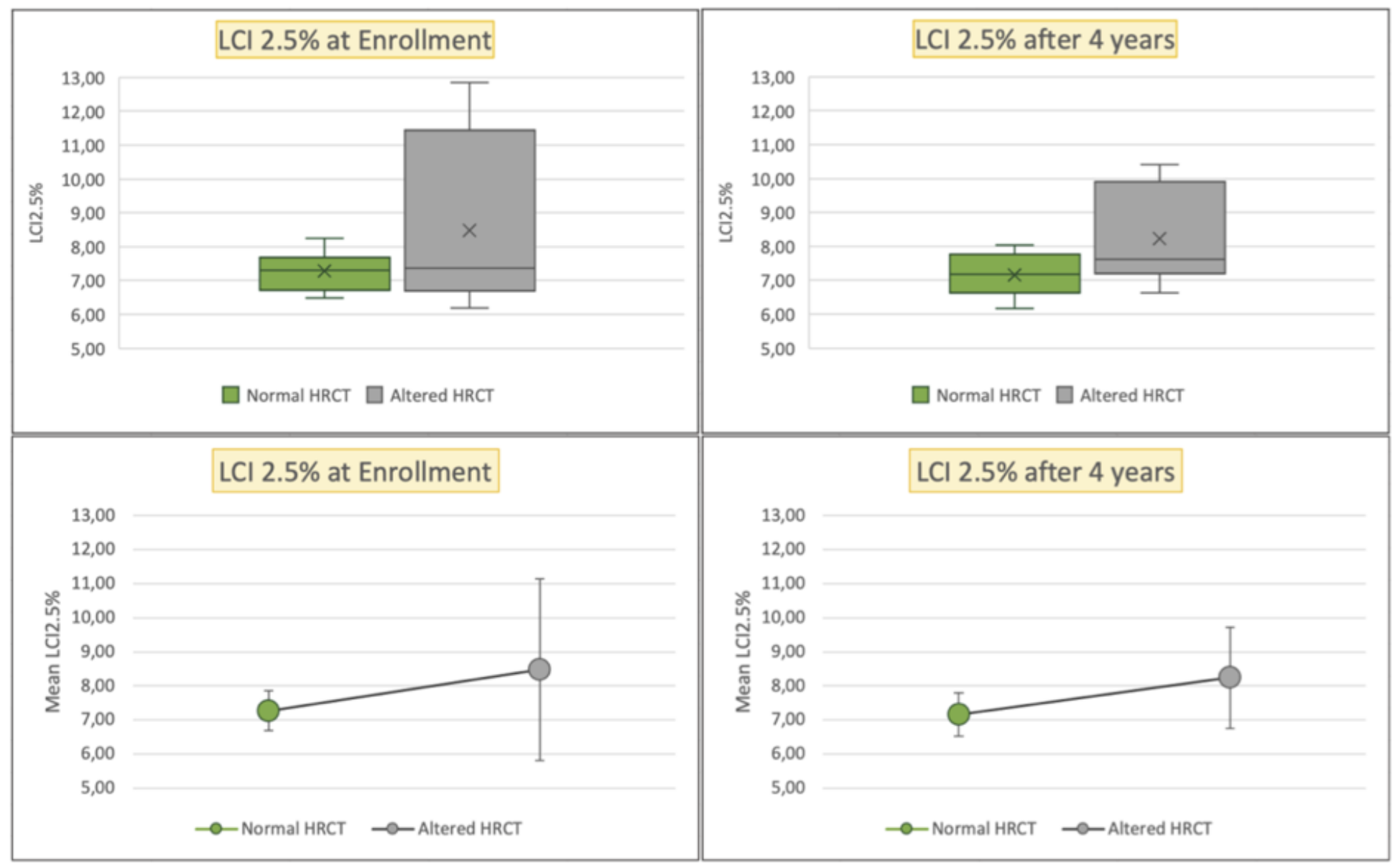

\section{Figure 5}

Box plots (above), Mean and Standard Deviation (below) of LCl at $2.5 \%$ of gas initial concentration respectively for patients with normal HRCT (green) or altered HRCT (gray), at enrollment (left) and at the HRCT control (right). LCl 2.5\%, Lung Clearance Index at $2.5 \%$ of gas initial concentration; HRCT, High Resolution Computed Tomography. 


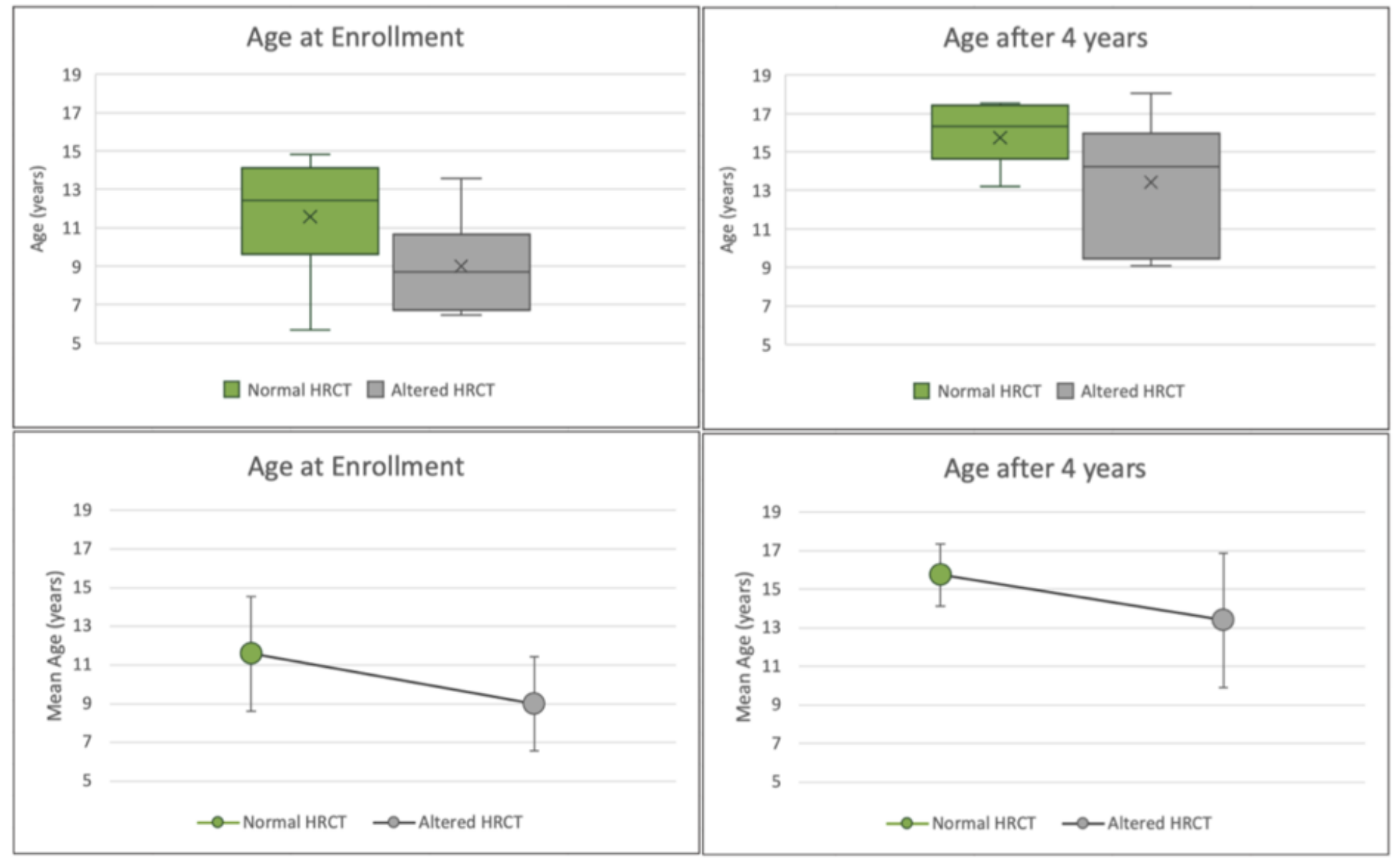

\section{Figure 6}

Box plots (above), Mean and Standard Deviation (below) of the age in years respectively for patients with normal HRCT (green) or altered HRCT (gray) at enrollment (left) and at the HRCT control (right). HRCT, High Resolution Computed Tomography. 